\section{Triptantrina: da Natureza ao Laboratório}

$>$

Daniela Pinheiro

Pedro Brandão

J. Sérgio Seixas de Melo Marta Pineiro*

\begin{abstract}
Tryptanthrin: from Nature to the Laboratory. Tryptanthrin is an indoloquinazoline alkaloid, present in several natural sources, particularly in plants. Used since ancient times for its anti-inflammatory, antipyretic and analgesic activities, today its biological properties continue to be subject of intense study. The interest in the development of various applications of tryptanthrin has stimulated the growth of several synthetic pathways and the in-depth study of its unique photophysical, photochemical and redox properties.

Tryptanthrin is an example of the many natural products with unique properties and relevant biological activity that demonstrates the importance of preserving natural sources, as guardians of knowledge.
\end{abstract}

\begin{abstract}
A triptantrina é um alcaloide indoloquinazolínico, presente em diversas fontes naturais, especialmente em diversas espécies vegetais. Utilizada desde a antiguidade pela sua atividade antiinflamatória, antipirética e analgésica, as suas propriedades biológicas continuam a ser alvo de intenso estudo. A investigação das diversas aplicações da triptantrina impulsionou o desenvolvimento de vários métodos de síntese e o estudo aprofundado das suas propriedades, nomeadamente fotofísicas, fotoquímicas e redox. A triptantrina é um exemplo das muitas moléculas presentes na natureza, com propriedades únicas e com atividade biológica relevante, o que demonstra a importância de se preservarem os recursos naturais.
\end{abstract}

\begin{abstract}
Introdução
As plantas são essenciais para a saúde do planeta e para a saúde dos humanos. Para além do seu papel fundamental na sustentabilidade ambiental e do seu valor nutricional, as plantas ou os seus extratos foram usados, durante muitos séculos, para prevenir ou curar doenças. Os extratos de plantas foram, e continuam a ser, fonte de compostos biologicamente ativos e, portanto, muitos dos medicamentos disponíveis contêm fitoquímicos ou análogos estruturais, produzidos sinteticamente para obter novas entidades químicas com a eficácia desejada. As plantas, contudo, continuam a ser uma biblioteca química infinita. A biodiversidade e a fitossanidade são por essa razão indispensáveis para preservar essa fonte quase inesgotável de compostos com atividade biológica.

A triptantrina é um exemplo de um produto natural com elevado interesse farmacológico. A utilização, na medicina tradicional, de extratos de plantas contendo triptantrina com efeito anti-inflamatório, antipirético e analgésico, impulsionou o interesse por esta molécula, pelo que a sua extração, caracterização estrutural, síntese e utilização continua a ser uma área de investigação muito ativa.
\end{abstract}

\section{Estrutura e fontes naturais da triptantrina}

A triptantrina é um sólido de aspeto amarelo brilhante (Figura 1), cujo nome advém do aminoácido triptofano, sendo produzida pela levedura Candida lipolytica quando este se encontra presente no meio de cultura.

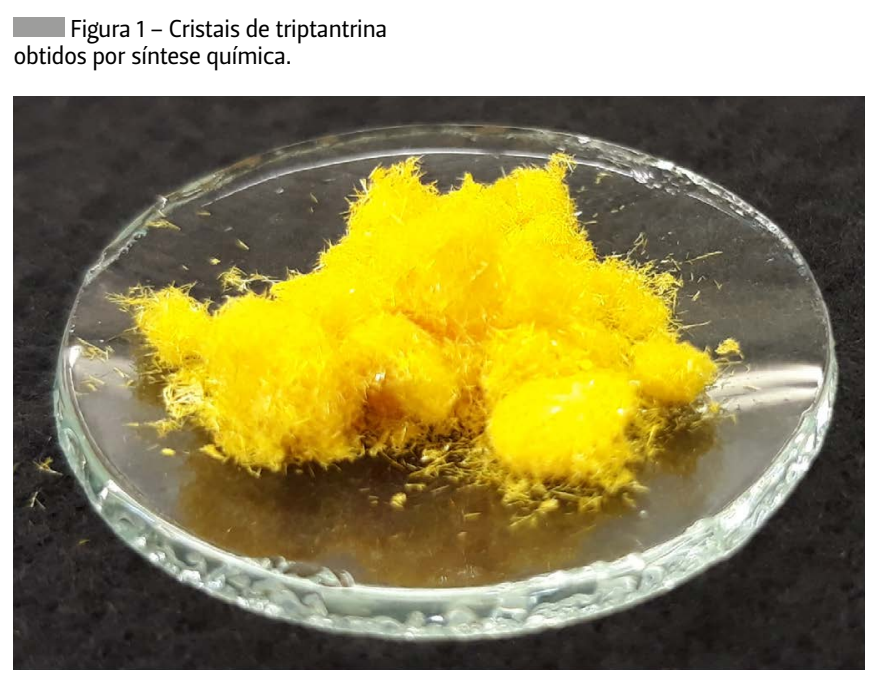


Em 2022 celebrar-se-á o 200. aniversário da síntese da triptantrina, dado que a sua preparação foi descrita pela primeira vez em 1822 [1]. Curiosamente, a estrutura só foi descoberta quase 100 anos depois, em 1915 [2], e só muito posteriormente confirmada por cristalografia de raios-X (1974). Citando os autores, "Tryptanthrin is an antibiotic isolated from the yeast Candida lipolytica. Crystallographic analysis was carried out in order to determine its structure as the preliminary chemical and spectroscopic (u.v., i.r., and n.m.r.) evidence was not sufficient for this purpose" [3].

A triptantrina, que de acordo com a nomenclatura IUPAC é designada por 6,12-di-hidro-6,12-dioxoindolo-[2,1-b]-quinazolina, é um alcaloide indoloquinazolínico, que resulta de um anel de quinazolina fundido com um indole e grupos carbonilo nas posições 6 e 12 (Figura 2).

A triptantrina tem sido isolada de diversas fontes naturais, incluindo plantas, fungos, bactérias e, curiosamente, também em mamíferos (nomeadamente da urina do elefante asiático, Elephas maximus, e do líquido do saco da asa do morcego Saccopteryx bilineata) [4]. De entre as plantas tradicionalmente usadas para produzir corantes, destacam-se as espécies Isatis tinctoria, Polygonum tinctorium, Strobilanthes cusia (índigo Assam), Strobilanthes formosanus (índigo naturalis) e Wrightia tinctoria [4-7]. Considerando

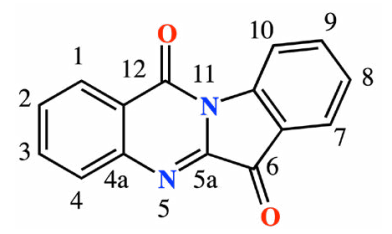

Figura 2 - Estrutura da triptantrina e respetiva numeração de acordo com a nomenclatura da IUPAC. estas fontes naturais, torna-se evidente a existência de uma relação entre a triptantrina e o índigo, um corante icónico usado desde tempos ancestrais até aos nossos dias, por exemplo no processo de tingimento da ganga $[8,9]$. Apesar da diversidade de fontes de obtenção natural, a triptantrina não é abundante na Natureza o que tem conduzido ao desenvolvimento de diversos métodos sintéticos.

\section{Síntese da triptantrina}

A triptantrina pode ser obtida através de diversas metodologias sintéticas, que foram compiladas por diversos autores $[4,10]$, e que podem ser estruturadas em três blocos, apresentados no Esquema 1: i) Reações de condensação para a construção do núcleo de quinazolina, i.e., condensação da isatina ou do oxindole, portador do núcleo de indole, com derivados do ácido antranílico (em particular o anidrido isatóico) conduzindo à formação do anel de quinazolina; ii) Reações de condensação e ciclização para construção do núcleo de quinazolina e indole, i.e, condensação de anéis de benzeno substituídos conduzindo à formação do anel de quinazolina e subsequente ciclização para formação do indole e iii) Reações de oxidação, entre as quais se destaca a oxidação do índigo, que se transforma em isatina e anidrido isatóico permitindo, através de condensação in situ, a formação da triptantrina. Há, ainda, alguns exemplos de quinazolinas substituídas que por ciclização originam a triptantrina (Esquema 1).
Reações de condensação Formação do anel de Quinazolina

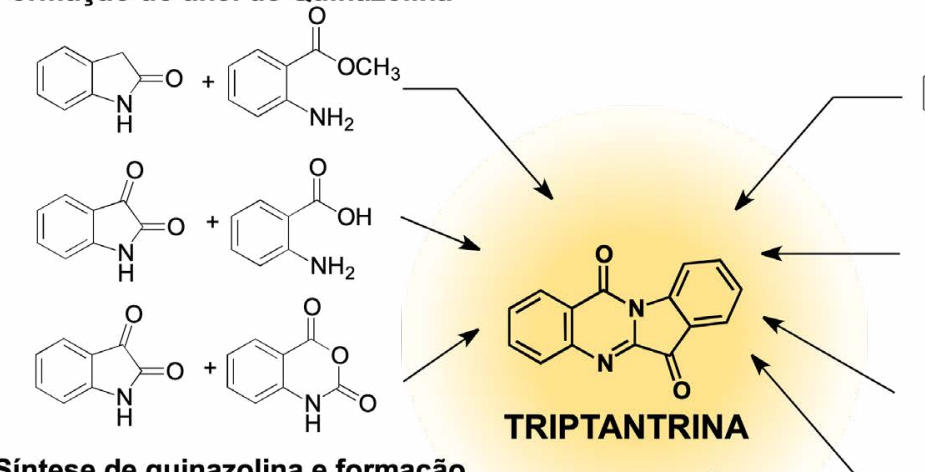

Síntese de quinazolina e formação do anel de indole por ciclização

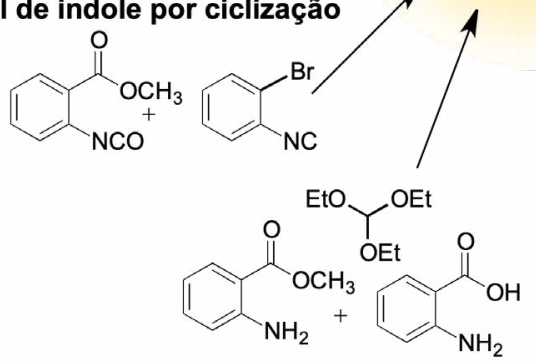<smiles>O=C(O)c1ccccc1-n1cnc2ccccc2c1=O</smiles>

Reações de oxidação<smiles></smiles><smiles>CC1=Nc2ccccc2C(=O)C1c1ccccc1</smiles>

Ciclização Formação do indole

Esquema 1 - Estratégias para a síntese de triptantrina. 
Desde que em 1892 O'Neill descreveu a primeira síntese de triptantrina por meio da oxidação do índigo com $\mathrm{KMnO}_{4}[11]$, apenas foram descritos alguns métodos oxidativos para a síntese de triptantrina. Estes incluem a oxidação do índigo usando ozono, com baixos rendimentos [12], e a oxidação da isatina com oxidantes fortes, como $\mathrm{KMnO}_{4}$ ou POCl$[3[13,14]$. Num estudo mais recente, e de forma inesperada, a triptantrina foi obtida como produto principal da reação de índigo com um di-iodoalcano na presença de $\mathrm{NaH}$ em $N, N^{\prime}$-dimetilformamida (DMF) sob irradiação de micro-ondas (MW, do inglês microwave). Da análise de diferentes condições reacionais concluiu-se que o sistema I/ $/$ NaH/DMF atua como agente oxidante. De facto, a DMF é a fonte de oxigénio que permite transformar índigo em isatina e anidrido isatóico in situ, os quais, após condensação, dão lugar à formação de triptantrina (Esquema 2). 0 mesmo sistema, $\mathrm{I}_{2} / \mathrm{NaH} / \mathrm{DMF}$, revelou ser eficaz na síntese da triptantrina a partir de isatina, promovendo a oxidação in situ a anidrido isatóico e posterior condensação [15]. A DMF, um solvente polar aprótico de elevado ponto de ebulição, para além da sua utilização como solvente, também possui a capacidade de servir como bloco de construção para várias unidades, nomeadamente grupos carbonilo, metilo ou dimetilamina [16]. A sua capacidade de atuar como fonte de oxigénio em reações de oxidação é menos comum $[17,18]$. No entanto, neste caso, permite sintetizar a triptantrina e seus derivados usando condições de reação moderadas e com elevados rendimentos. De facto, tal é particularmente evidente quando comparados com os métodos descritos anteriormente usando índigo como material de partida, e ao mesmo tempo sem necessidade de recorrer a oxidantes fortes ou a montagens laboratoriais complexas [15]. 0 desenvolvimento de métodos sintéticos aumenta a disponibilidade da triptantrina e dos seus derivados, permitindo o estudo mais diversificado das suas propriedades e o desenvolvimento de aplicações envolvendo estes compostos.

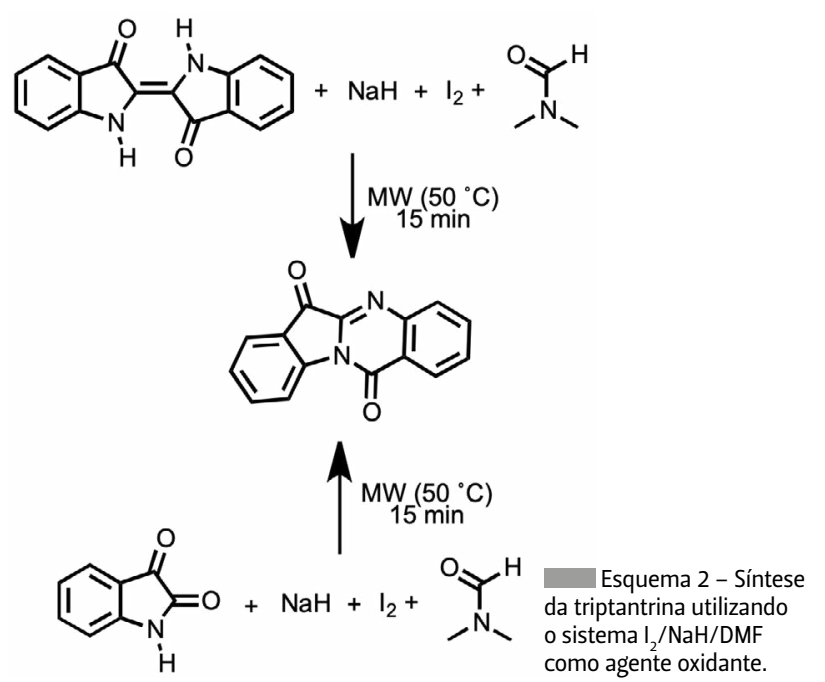

Triptantrina no estado eletrónico excitado As propriedades espectroscópicas da triptantrina são altamente dependentes do solvente. Em acetonitrilo, solvente polar aprótico, a triptantrina apresenta um espectro de absorção com uma banda na região do visível (comprimento de onda máximo de absorção, $\lambda_{\text {abs }}$, de 394 nm) e um espectro de emissão de fluorescência com uma banda com comprimento de onda máximo, $\lambda_{\text {fluo }}$, de $513 \mathrm{~nm}$. Em solventes não polares e apróticos, por exemplo, $n$-pentano, a banda de absorção e a banda de emissão sofrem um desvio hipsocrómico $\left(\lambda_{\text {abs }}=390\right.$ $\left.\mathrm{nm} ; \lambda_{\text {fluo }}=506 \mathrm{~nm}\right)$. Contudo, em solventes polares próticos, e.g., metanol, o desvio hipsocrómico da banda de absorção é acompanhado por um desvio batocrómico da banda de fluorescência $\left(\lambda_{\text {abs }}=389 \mathrm{~nm} ; \lambda_{\text {fluo }}=552 \mathrm{~nm}\right.$ ). Em termos fotofísicos, o facto da triptantrina apresentar baixos rendimentos quânticos de fluorescência e curtos tempos de vida de fluorescência ( $\varphi_{F}$ e $\tau_{F}$, respetivamente), com valores de $\varphi_{F}=0,008$ e $\tau_{F}=499$ ps em acetonitrilo e $\varphi_{F}=0,002$ e $\tau_{F}=146$ ps em metanol, tornam-na semelhante ao índigo. No entanto, as comparações terminam aqui. Enquanto que no índigo o rendimento de formação de estado tripleto e de formação de oxigénio singleto é inferior a 0,01\% (a conversão interna associada a um mecanismo de transferência de protão no estado excitado domina em 99,99\% o processo de desativação) [19], no caso da triptantrina o rendimento quântico de formação de oxigénio singleto é, em acetonitrilo, de 0,77 . De facto, ao contrário do índigo, na triptantrina a proximidade das bandas de fluorescência e fosforescência indicam que os estados singleto excitado $\left(\mathrm{S}_{1}\right)$ e estado tripleto excitado $\left(T_{1}\right)$ têm energias muito próximas, o que suporta a eficácia do cruzamento interssistemas do estado $\mathrm{S}_{1}$ para o estado $\mathrm{T}_{1}$ (Esquema 3).

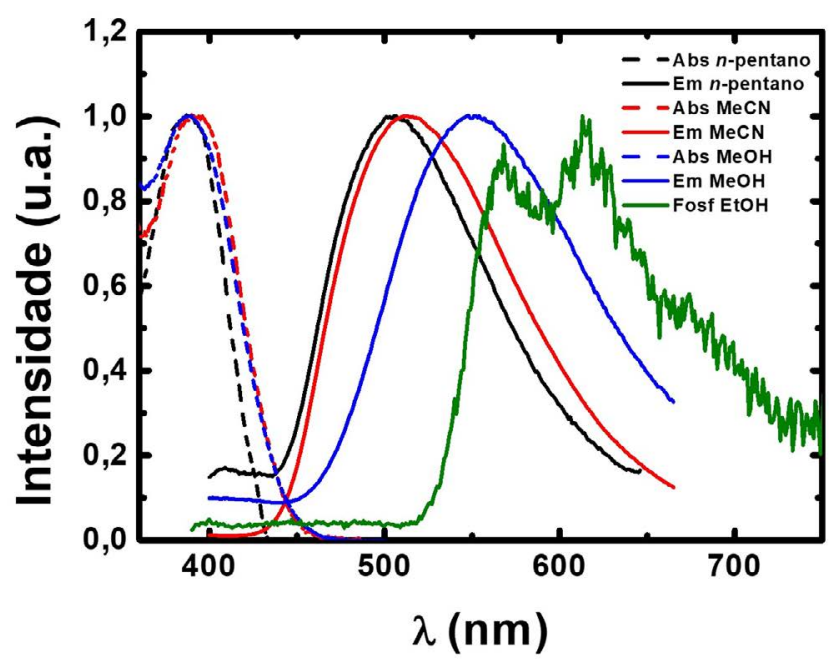

Figura 3 - Espectros de absorção e emissão de fluorescência da triptantrina em $n$-pentano, acetonitrilo e metanol à temperatura ambiente e espectro de emissão de fosforescência em etanol a $77 \mathrm{~K}$. Abs, absorção; Em: emissão de fluorescência; Fosf: emissão de fosforescência. 
Estudos aprofundados de fluorescência resolvida no tempo e de absorção do estado transiente na escala de femtossegundos, complementados com cálculos teóricos através da teoria do funcional da densidade dependente do tempo (TDDFT, do inglês Time-dependent density-functional theory), permitiram racionalizar as propriedades fotofísicas da triptantrina e propor o diagrama de níveis de energia (Esquema 3) com os estados teoricamente previstos e as transições observadas experimentalmente em acetonitrilo. Após a absorção de luz, o canal de desativação principal é uma emissão não radiativa para um estado $\Pi, \Pi^{*}$ (LE, do inglês Local Excited), diferente do estado $\mathrm{n}, \Pi^{*}$ (CT, do inglês Charge Transfer) que dá lugar à formação do estado tripleto, fracamente emissivo por fosforescência, mas extremamente eficaz na sensibilização do oxigénio [20]. Esta propriedade permite a utilização da triptantrina em aplicações biológicas relacionadas com a produção de ROS (do inglês Reactive Oxygen Species).

\section{Propriedades redox}

Além das propriedades fotofísicas interessantes, a triptantrina também tem capacidade de realizar reversivelmente processos de oxidação e redução. 0 voltamograma da triptantrina obtido em acetonitrilo utilizando o hexafluorofosfato de tetrabutilamónio $\left(\mathrm{NBu}_{4} \mathrm{PF}_{6}\right)$ como eletrólito de suporte (Figura 4) apresenta duas ondas reversíveis com picos catódicos e anódicos separados por aproximadamente 0,60 V, indicando duas transferências de um eletrão. A transformação a -1,12 V atribui-se à transferência de um eletrão para o oxigénio do grupo carbonilo do anel de cinco átomos, enquanto que num potencial mais negativo, $-1,69 \mathrm{~V}$, ocorre a transferência de um eletrão para o grupo carbonilo do anel de seis átomos (Esquema 4) [21]. Muitas das potenciais aplicações farmacológicas parecem estar associadas às propriedades redox deste composto $[21,22]$.

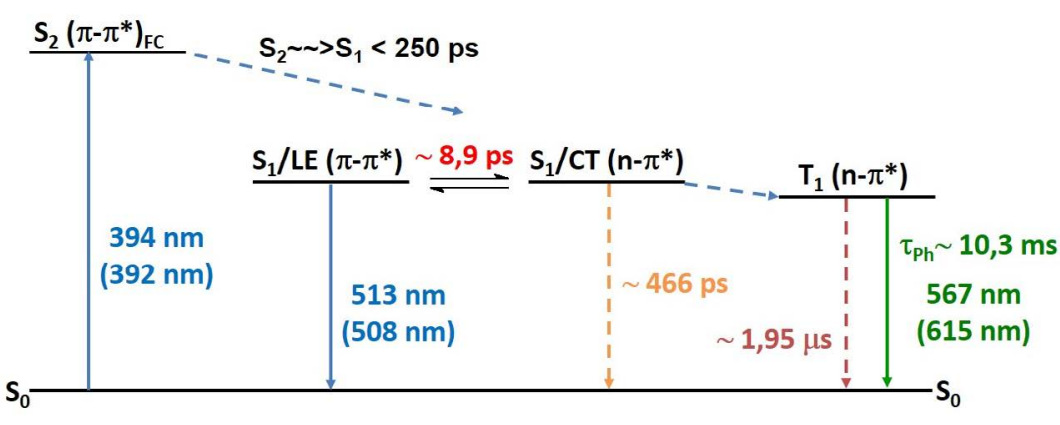<smiles>O=C1c2ccccc2-n2c1nc1ccccc1c2=O</smiles><smiles>Oc1c2ccccc2n2c(O)c3ccccc3nc12</smiles>

Esquema 3 - Diagrama de níveis de energia calculados por TDDFT e determinados experimentalmente. Os valores entre parêntesis correspondem às transições previstas pelos cálculos TDDFT.

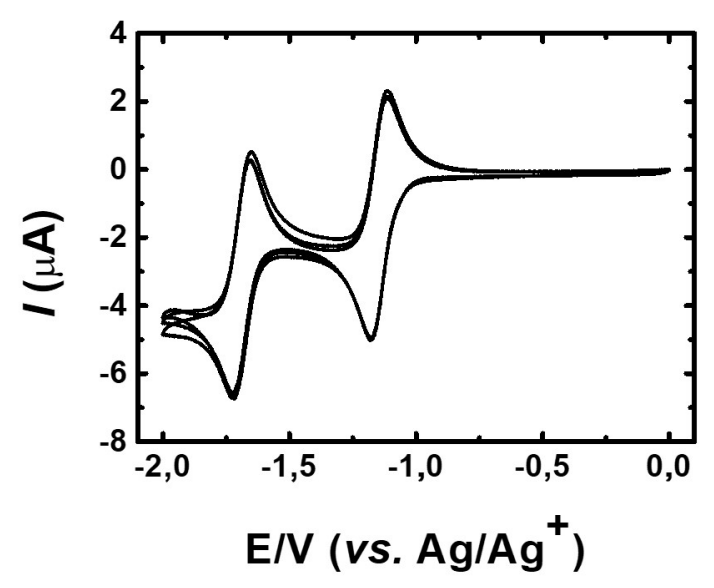

Figura 4 - Voltamograma cíclico de uma solução 1,0 mM de triptantrina em acetonitrilo $\left(0,1 \mathrm{M} \mathrm{NBu}_{4} \mathrm{PF}_{6}\right)$, saturada com $\mathrm{N}_{2}, \mathrm{v}=50 \mathrm{mV} \mathrm{s}^{-1}$. 


\section{Aplicações biológicas}

Como se referiu na introdução, os extratos naturais contendo triptantrina são utilizados desde a antiguidade pelas suas propriedades terapêuticas, sendo conhecida desde longa data a sua capacidade de atuar como anti-inflamatório, antipirético e analgésico [23,24]. Para além destas, a triptantrina é ainda conhecida pela sua atividade como agente anti-infecioso, demonstrando atividade contra fungos, parasitas (incluindo-se nestes os agentes causadores da malária e da leishmaniose), bactérias (nomeadamente a Mycobacterium tuberculosis), anticancerígeno e anti-inflamatório [25-31].

Recentemente, a triptantrina e o seu derivado com um grupo oxima na posição 6 foram estudados como anti-inflamatórios para o tratamento da artrite reumatoide [32]. Foi demonstrada a capacidade da triptantrina atuar como regulador da inflamação vascular através da regulação do TLR3 (do inglês Toll-like receptor 3) [33]. A triptantrina também foi identificada, no extrato de índigo naturalis, como o composto com maior efeito antiproliferativo em cinco tipos de células identificadas em lesões de psoríase [34], e revelou ser um agente antimicótico eficaz como adjuvante e para a prevenção de reinfeções fúngicas [35]. Foi também sintetizado um novo derivado da triptantrina mais solúvel em água, o mostotrin, que demonstrou ter capacidades promissoras como agente antimicrobiano e antitumoral [36]. A triptantrina e doze novos derivados revelaram ter elevada dupla potência inibitória da indolamina 2,3-dioxigenase 1 (ID01) e da triptofano 2,3-dioxigenase (TDO), promissores alvos no desenvolvimento de novos fármacos devido ao seu potencial no tratamento de patologias como o cancro e doenças neurodegenerativas [37].

Em 2020, ano que será sem dúvida recordado pela situação pandémica causada pela transmissão do vírus SARS-CoV-2, o extrato de Strobilanthes cusia (Kuntze), utilizado na medicina tradicional chinesa para o tratamento de infeções respiratórias causadas por vírus (que contém entre outros compostos a triptantrina), foi avaliado como antivírico contra o coronavírus humano HCoV-NL63 [38]. Neste estudo conclui-se que a triptantrina altera especificamente a estrutura antigénica das proteínas spike e, para além disso, parece atuar em diferentes estágios da replicação viral através do bloqueio da síntese de ARN viral e atividade de uma protease papain-like. Uma nota importante a salientar é a de que a proteína spike HCoV-NL63 apresenta uma sequência estruturalmente similar ao SARS-CoV e ao SARS-CoV-2. Assim, a triptantrina, exibindo ação antiviral e impedindo a replicação pós-infeção, constitui um bom ponto de partida para o desenvolvimento de novos fármacos a aplicar na terapêutica de infeções causadas por coronavírus humanos.

\section{Conclusões}

Pelo exposto, é evidente que o estudo das propriedades da triptantrina, dos seus derivados e dos extratos naturais que a contêm continuará a ser um alvo de interesse de diversos grupos de investigação devido, muito em particular, às suas potenciais aplicações biológicas. A triptantrina é apenas um exemplo dos muitos compostos com propriedades únicas que a Natureza sintetiza. A preservação das fontes Naturais, a biodiversidade e a fitossanidade são fundamentais para manter a biblioteca química que a Natureza nos oferece, a qual, como todas as bibliotecas, é guardiã do conhecimento e promotora do saber.

\section{Agradecimentos}

Este trabalho foi suportado pelo projeto "SunStorage - Harvesting and storage of solar energy" referência POCl-01-0145-FEDER-016387 e pelo projeto "Hylight" (n. $\left.{ }^{\circ} 031625\right)$ 02/SAICT/2017, PTDC/QUI-QFI/31625/2017, ambos financiados pelo Fundo Europeu de Desenvolvimento Regional (FEDER), através do Programa Operacional Factores de Competitividade (COMPETE) 2020 e Programa Operacional para a Competitividade e Internacionalização (OPCI), e por fundos nacionais através da Fundação para a Ciência e a Tecnologia (FCT). 0 centro de Química de Coimbra é financiado pela FCT através do projeto UID/QUI/00313/2019. D. Pinheiro agradece o financiamento à FCT (SFRH/BD/74351/2010) tal como P. Brandão (PD/BD/128490/2017 - CATSUS FCT-PhD Program). D. Pinheiro também agradece ao projeto "SunStorage - Harvesting and storage of solar energy" pelo financiamento de uma bolsa de investigação.

\section{Referências}

[1] P. Siedel, 1822 BASF, Ludwiǵshafen 1983, no qual se descreve a preparação da triptantrina em 1822 por Dumas (J. Pharm. VIII, 1822, 377). Não foi possível aceder à referência original.

[2] P. Friedländer, N. Roschdestwensky, Chem. Ber. 1915, 48, 1841. DOI: 10.1002/ cber.19150480279.

[3] W. Fedeli, F. Mazza, J. Chem. Soc., Perkin Trans. 2 1974, 1621-1623. DOl 10.1039/P29740001621.

[4] A. M. Tucker, P. Grundt, Arkivoc 2012, i, 546-569. D0I: 10.3998/ ark.5550190.0013.113.

[5] Y. Jahng, Arch. Pharm. Res. 2013, 36, 517-535. DOI: 10.1007/s12272-0130091-9.

[6] U. A. Kshirsagar, Org. Biomol. Chem. 2015, 13, 9336-9352. DOI: 10.1039/ c5ob01379h.

[7] R. C. Garcellano, S. G. A. Moinuddin, R. P. Young, M. Zhou, M. E. Bowden, R. S. Renslow, Y. Yesiltepe, D. G. Thomas, S. M. Colby, C. D. Chouinard, G. Nagy, I. K. Attah, Y. M. Ibrahim, R. Ma, S. G. Franzblau, N. G. Lewis, A. M. Aguinaldo, J. R. Cort, J. Nat. Prod. 2019, 82, 440-448. DOI: 10.1021/acs.jnatprod.8b00567.

[8] N. Gaboriaud-Kolar, S. Nam, A.-L. Skaltsounis, "Progress in The Chemistry of Organic Natural Products", Springer, London, 2014.

[9] J. Seixas de Melo, M. Barroso, Química 2001, 81, 66-69. DOl: 10.52590/ M3.P605.A3000983.

[10] R. Kaur, S. K. Manjal, R. K. Rawal, K. Kumar, Bioorg. Med. Chem. 2017, 25, 4533-4552. DOI: 10.1016/j.bmc.2017.07.003. 
[11] C. O'Neill, Chem. News 1892, 65, 124. (E-book livre acesso em play.google.com/books/reader?id=p5WEAAAAYAAJ\&hl=ptPT\&printsec $=$ frontcover \&pg $=$ GBS.PA124).

[12] H. Machemer, Ber. Dtsch. Chem. Ges. B. 1930, 63B, 1341.

[13] T. V. Moskovkina, M. V. Denisenko, A. I. Kalinovskii, V. A. Stonik, Rus. J. Org. Chem. 2013 , 49, 1740-1743. DOl: 10.1134/S1070428013120051.

[14] T. V. Moskovkina, A. I. Kalinovskii, V. V. Makhan'kov, Rus. J. Org. Chem. 2012 48, 123-126. DOI: 10.1134/S1070428012010204.

[15] P. Brandão, D. Pinheiro, J. S. Seixas de Melo, M. Pineiro, Dyes Pigm. 2020 , 173, 107935-107939. DOl: 10.1016/j.dyepig.2019.107935.

[16] S. Ding, N. Jiao, Angew. Chem. Int. Ed. 2012, 51, 9226-9237. DOI: 10.1002/ anie.201200859.

[17] J. Muzart, Tetrahedron 2009, 65, 8313-8323. D0I: 10.1016/j. tet.2009.06.091.

[18] C. H. Jin, H. Y. Lee, S. H. Lee, I. S. Kim, Y. H. Jung, Synlett 2007,17, 2695 2698. DOI: 10.1055/s-2007-991081.

[19] J. S. Seixas de Melo, H. D. Burrows, C. Serpa, L. G. Arnaut, Angew. Chem. Int. Ed. 2007, 46, 2094-2096. DOI: 10.1002/anie.200604679.

[20] D. Pinheiro, M. Pineiro, J. Pina, P. Brandão, A. M. Galvão, J. S. Seixas de Melo Dyes Pigm. 2020, 175, 108125-108135. DOI: 10.1016/j.dyepig.2019.108125.

[21] A. K. Bhattacharjee, D. J. Skanchy, B. Jennings, T. H. Hudson, J. J. Brendle, K. A. Werbovetz, Bioorg. Med. Chem. 2002, 10, 1979-89. DOI: 10.1016/s0968 0896(02)00013-5.

[22] A. A. Klimovich, A. M. Popov, O. N. Krivoshapko, Y. P. Shtoda, A. V. Tsybulsky, Biophysics 2017, 62, 588-594. DOl: 10.1134/S0006350917040108.

[23] H. Yu T.-n. Li, Q. Ran, Q.-W. Huang, J. Wang, J. Ethnopharmacol. 2021, 265, 113325. DOI: 10.4314/njbas.v25i1.6.

[24] J. Speranza, N. Miceli, M. F. Taviano, S. Ragusa, I. Kwiecień, A. Szopa, H. Ekiert, Plants (basel) 2020, 9, 298. DOI: 10.3390/plants9030298.

[25] R. Kaur, S. K. Manjal, R. K. Rawal, K. Kumar, Bioorg. Med. Chem. 2017, 25, 4533-4552. DOl: 10.1016/j.bmc.2017.07.003.

[26] P. I. Deryabin, T. V. Moskovkina, L. S. Shevchenko, A. I. Kalinovskii, Russ. J.

\section{Daniela Pinheiro}

Universidade de Coimbra, Departamento de Química.

Centro de Química de Coimbra (CQC). Daniela Pinheiro é Doutorada em Química, ramo de especialização em Fotoquímica pela Universidade de Coimbra (2021). 0 seu foco de trabalho centra-se na síntese, estudos fotoquímicos e eletroquímicos de derivados da triptantrina e índigo e nas novas aplicações dessas moléculas com história. dpinheiro@qui.uc.pt

ORCID.org/0000-0001-5897-6020

\section{J. Sérgio Seixas de Melo}

Universidade de Coimbra, Departamento de Química.

Centro de Química de Coimbra (CQC).
Doutoramento em Química-Física (IST-UL, 1996) e Agregação em Química (UC, 2012). Prof. Associado do DQ da Universidade de Coimbra e investigador do Centro de Química de Coimbra e do Coimbra Laser Lab. As suas atividades de investigação centram-se na fotoquímica e fotofísica, abrangendo a caracterização dos estados excitados de moléculas com aplicações em DSSC, RFB e OLEDs e no domínio da Química e Arte, incluindo o estudo de moléculas icónicas como o índigo. $\mathrm{H}$-index 46 resultante de mais de 6600 citações, mais de 200 artigos e 15 capítulos de livros. É responsável pelo grupo e laboratórios de Fotoquímica da UC (photochemistry.pt). sseixas@ci.uc.pt ORCID.org/0000-0001-9708-5079
Org. Chem. 2017, 53, 418-422. DOI: 10.1134/S1070428017030174.

[27] M. J. Novak, J. Clayton Baum, J. W. Buhrow, J. A. Olson, Surf. Sci. 2006, 600, L269-L73. DOI: 10.1016/j.susc.2006.07.036.

[28] J. Kawakami, N. Matsushima, Y. Ogawa, H. Kakinami, A. Nakane, H. Kitahara, M. Nagaki, S. Ito. Trans. Mat. Res. Soc. Japan. 2011, 36, 603-606. DOI: 10.14723/tmrsj.36.603.

[29] A. S. Filatov, N. A. Knyazev, S. V. Shmakov, A. A. Bogdanov, M. N. Ryazantsev, A. A. Shtyrov, G. L. Starova, A. P. Molchanov, A. G. Larina, V. M. Boitson, A. V. Stepakov, Synthesis 2019, 51, 713-29. D0l: 10.1055/s-0037-1611059.

[30] R. Amara, H. Awad, D. Chaker, B.-A. Ghenia, F. Lassagne, W. Erb, F. Chevallier, T. Roisnel, V. Dorcet, Z. Fajloun, J. Vidal, F. Monglin, Eur. J. Org. Chem. 2019, 5302-5312. DOI: 10.1002/ejoc.201900352.

[31] M. Hamburger, Phytochem. Rev. 2002, 1, 333-344. DOI: 10.1023/A:1026095608691.

[32] L. N. Kirpotina, I. A. Schepetkin, D. Hammaker, A. Kuhs, A. I. Khlebnikov, M. T. Quinn, Front. Pharmacol. 2020, 11, 1145. DOI: 10.3389/fphar.2020.01145.

[33] S. Kawaguchi, H. Sakuraba, H. Kikuchi, N. Numao, T. Asari, H. Hiraga, J. Ding, T. Matsumiya, K. Seya, S. Fukuda, T. Imaizumi, Mol. Immunol. 2020, 129, 32-38. DOI: 10.1016/j.molimm.2020.11.003.

[34] H.-M. Cheng, Y.-Z. Kuoc, C.-Y. Chang, C.-H. Chang, W.-Y. Fang, C.-N. Chang S.-C. Pan, J.-Y. Ling, L.-W. Wu, J. Ethnopharmacol. 2020, 255, 112760. DOl: 10.1016/j.jep.2020.112760.

[35] J. Hesse-Macabata, B. Morgner, P. Elsner, U.-C. Hipler, C. Wiegand, Sci. Rep. 2020, 5, 1863. DOI: 10.1038/s41598-020-58773-2.

[36] A. Popov, A. Klimovich, O. Styshova, T. Moskovkina, A. Shchekotikhin, N. Grammatikova, L. Dezhenkova, D. Kaluzhny, P. Deriabin, A. Gerasimenko, A. Udovenko, V. Stonik, Int. J. Mol. Med. 2020, 46, 1335-1346. DOI: $10.3892 \% 2$ Fijmm.2020.4693.

[37] Y. Li, S. Zhang, R. Wang, M. Cui, W. Liu, Q. Yang, C. Kuang, Bioorg. Med. Chem. Lett. 2020, 30, 127159. DOI: 10.1016/j.bmcl.2020.127159.

[38] Y.-C. Tsai, C.-L. Lee, H.-R. Yen, Y.-S. Chang, Y-P. Lin, S.-H. Huang, C.-W. Lin, Biomolecules 2020, 10, 366. DOI: 10.3390/biom10030366.

\section{*Marta Pineiro}

Universidade de Coimbra, Departamento de Química.

Centro de Química de Coimbra (CQC). Licenciatura em Química (Universidade de Santiago de Compostela) e Doutoramento em Química (Universidade de Coimbra 2002), onde é Professora Auxiliar. Os interesses de investigação centram-se na Química Verde, na síntese assistida por micro-ondas, na mecanoquímica e no desenvolvimento de processos sustentáveis para a síntese de heterociclos com propriedades fotofísicas únicas e/ou com potencial aplicação biológica. Publicou mais de 70 artigos científicos e sete capítulos de livros.

mpineiro@qui.uc.pt ORCID.org/0000-0002-7460-3758

\section{Pedro Brandão}

Universidade de Coimbra, Departamento de Química.

Centro de Química de Coimbra (CQC). LAQV-REQUIMTE. Departamento de Química, Universidade de Évora.

Pedro Brandão é Mestre em Ciências Farmacêuticas pela FFUP (2011) e frequenta o Doutoramento em Química (ramo de Catálise e Sustentabilidade), sendo bolseiro do programa doutoral CATSUS (Universidade de Coimbra, Universidade de Évora e Instituto Superior Técnico). o seu foco de trabalho centra-se na descoberta de novas moléculas com potencial atividade farmacológica, usando reações multicomponente e metodologias sustentáveis. pbrandao@qui.uc.pt ORCID.org/0000-0002-1455-7470 